\title{
The position of the inferior vena cava-right atrium junction on simple radiography: implication for optimal veno-venous extracorporeal membrane oxygenation cannula tip position
}

\author{
Dong Su Kim ${ }^{1}$, Yun Gyu Song ${ }^{1}$, Yong Hwan Kim ${ }^{2}$, Moon Ok Lee ${ }^{3}$ \\ ${ }^{1}$ Department of Radiology, ${ }^{2}$ Department of Emergency Medicine, ${ }^{3}$ Department of Anesthesia and Pain Medicine, Samsung Changwon Hospital, \\ Sungkyunkwan University School of Medicine, Changwon, Korea \\ Contributions: (I) Conception and design: DS Kim, MO Lee; (II) Administrative support: YG Song, MO Lee; (III) Provision of study materials or \\ patients: YG Song, MO Lee; (IV) Collection and assembly of data: YH Kim, DS Kim; (V) Data analysis and interpretation: DS Kim, YH Kim; (VI) \\ Manuscript writing: All authors; (VII) Final approval of manuscript: All authors. \\ Correspondence to: Moon Ok Lee, MD. Department of Anesthesia and Pain Medicine, Samsung Changwon Hospital, Sungkyunkwan University \\ School of Medicine, 158, Paryong-ro, Masanhoewon-gu, Changwon-si, Gyeongsangnam-do 51353, Korea. Email: lmo602@hanmail.net.
}

\begin{abstract}
Background: Extracorporeal membrane oxygenation (ECMO) is increasingly used. For effective oxygenation, position of cannula tip is important. The objective of this study is to identify the position of the inferior vena cava-right atrium junction (IRJ) using the vertebral body unit (VBU) and carina. This may be useful if applied to the assessment of ECMO cannula tip positions.

Method: A total of 182 patients who underwent chest computed tomography (CT) and supine chest radiography (CXR) between January 2017 and July 2018 were included, who had not previously received ECMO support. The position of the IRJ was evaluated using VBU and the efficacy of the VBU for locating the IRJ with the carina was analyzed.

Result: The mean distance (SD) from carina to IRJ was 79.2 (9.6) $\mathrm{mm}$ on chest CT. The mean distance in VBUs (SD) at the level of the carina was $22.3(1.5) \mathrm{mm}$ on chest CT and 23.3 (2.1) $\mathrm{mm}$ on CXR. The mean IRJ position was 3.6 VBUs below the carina on CT and 3.4 VBUs below on CXR with $95 \%$ limits of agreement between -0.05 and 0.5 .
\end{abstract}

Conclusions: The mean position of the IRJ was estimated to be 3.4 VBUs below the carina on supine CXR. This may be useful if applied to the assessment of ECMO cannula tip positions.

Keywords: Extracorporeal membrane oxygenation (ECMO); inferior vena cava (IVC); oxygenation; right atrium; vertebra

Submitted May 23, 2019. Accepted for publication Aug 27, 2019.

doi: $10.21037 /$ jtd.2019.09.10

View this article at: http://dx.doi.org/10.21037/jtd.2019.09.10

\section{Introduction}

Extracorporeal membrane oxygenation (ECMO) is increasingly used for patients with respiratory and/or cardiac failure (1). Among the factors affecting effective oxygenation in veno-venous $(\mathrm{V}-\mathrm{V}) \mathrm{ECMO}$, the amount of recirculation can be affected by cannula position (2). Therefore, adequate tip position of $\mathrm{V}-\mathrm{V}$ ECMO cannula is important. In the $\mathrm{V}-\mathrm{V}$ $\mathrm{ECMO}$ with classic feature, drainage cannula tip is positioned at or just distal to the inferior vena cava-right atrium junction
(IRJ) and a return cannula is positioned at the right atriumsuperior vena cava junction or within the right atrium (RA) (3). And this position is confirmed by echocardiography in general, which is operator-dependent and is inconvenient for the patients when performed (4).

Radiologic landmarks, an objective structure such as carina and spine have not been used to confirm optimal cannula tip position in ECMO circuits. Baskin and coworkers (5) proposed the use of vertebral body unit (VBU) 
for determining optimal central venous catheter tip position. A VBU was defined as distance between two adjacent vertebral bodies including intervertebral disc space (5). Therefore, the purpose of this study was to identify the position of the IRJ using the VBU and carina on simple radiography. And, this may be useful if applied to the assessment of ECMO cannula tip positions.

\section{Methods}

\section{Patients}

The institutional review board approved this retrospective study and written informed consents were waived. Between January 2017 and July 2018, 257 consecutive patients with suspected lung cancer underwent chest computed tomography (CT) and anteroposterior (AP) supine projection in chest radiography (CXR) were enrolled. The patients had not previously received ECMO support. The exclusion criteria were as follows: lung cancer patients with invasion of central vein or central airway $(n=19)$, patients with chest surgery $(n=31)$ and patients with spine surgery or severe kyphosis $(\mathrm{n}=25)$.

\section{Imaging acquisition}

Chest CT images were obtained using a 128-row detector CT scanner (Somatom Definition AS+; Siemens Medical Solutions, Forchheim, Germany). Chest CT was performed with the following protocols: $120 \mathrm{kVp}$, rotation time $0.5 \mathrm{sec}$, collimation $128 \mathrm{~mm} \times 0.6 \mathrm{~mm}$, pitch 1.3 . The $\mathrm{mAs}$ was automatically controlled according to the individual patient. Contrast enhancement was achieved by injection of $80 \mathrm{~mL}$ of iodinated contrast material $\left(350 \mathrm{mg}\right.$ iodine $\mathrm{mL}^{-1}$, Pamiray; Dongkook pharmaceutical, Seoul, Korea) followed by $20 \mathrm{~mL}$ saline flush (flow rate $2.5 \mathrm{~mL} / \mathrm{s}$ ). Automated bolus tracking was used in a region of interest within the descending aorta, with a signal attenuation trigger threshold of 100 Hounsfield units (HU) and a 15 s scan delay. CXR was performed in the supine position and was included the superior lung apex to below the costophrenic angles using the breath-hold technique with full inspiration (DigitalDiagnost; Philips Digital Radiography Solutions, Germany). The film to focus distances was $100 \mathrm{~cm}$ in supine position.

\section{Measurement}

All images were reviewed independently by two radiologists
(YG Song and DS Kim, who have 19 and 3 years of radiology experience, respectively), who were blinded to patient data. Measurements that differed by more than $3 \mathrm{~mm}$ were re-measured and then determined by consensus of two radiologists. The analysis was performed as averaging measurements obtained by the two radiologists.

Chest CT images were recalled from PACS (a Picture Archiving and Communications System, Marosis-Infinitt, Seoul, Korea) to a three-dimensional workstation (Aquarius intuition; Terarecon, San Francisco, CA, USA). On multiplanar reconstructed images, the following structures were identified: the anatomic IRJ, the carina, and VBU at the level corresponding to the carina.

The location of the IRJ is defined as the point or level which IVC and RA are connected (6) and the carina is the point or level at which the trachea divides into the right and left main bronchi. They are well-delineated in axial, reconstructed sagittal and coronal planes (Figure 1). A VBU was defined as distance between two adjacent vertebral bodies including intervertebral disc space. The VBU was measured from the inferior endplate of the vertebra at the carina level to the inferior endplate of the lower vertebra on chest CT and CXR (Figure 1).

The following measurements were obtained: distance from the carina to the IRJ, the VBU on chest CT, and the VBU on CXR. And then, the distance from the carina to the IRJ was measured in VBUs on both chest CT and CXR.

\section{Statistical analysis}

Results are presented as the median, mean (SD) and range. The Student's t-test or ANOVA was applied to compare variables between two or more groups. Correlations between the distance from the carina to the IRJ, the IRJ position in VBUs on CXR and other variables were analyzed using Pearson's correlation method. Inter-rater reliability between the two radiologists was assessed using the intra-class correlation coefficient. Variables found to be significant were selected and included in multiple linear regression analysis. The Bland-Altman plot was applied for the reproducibility of measurements measured on chest CT and CXR. All statistical analyses were performed using PASW software (version 18.0; SPSS, Chicago, IL, USA). Statistical significance was set at $\mathrm{P}<0.05$.

\section{Result}

Total 182 patients were included in our study. There were 

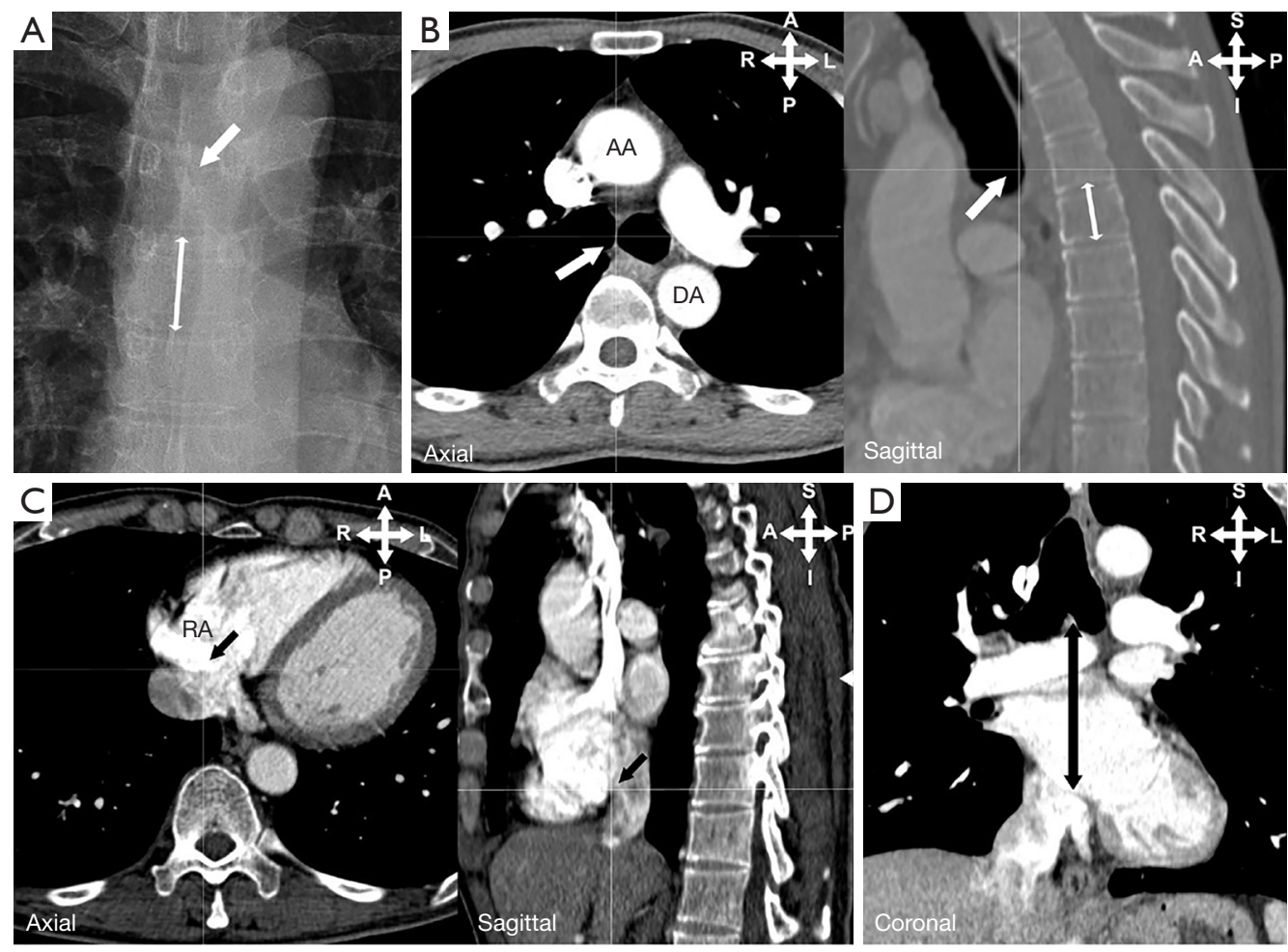

Figure 1 The carina and VBU are indicated by white single and white double-sided arrows. The IVC-RA junction and distance from carina to IVC-RA junction are indicated by black single arrow and black double-sided arrow. Anterior (A) to posterior $(\mathrm{P})$, right $(\mathrm{R})$ to left $(\mathrm{L})$, and superior (S) to inferior (I) orientations are shown. (A) Simple CXR; (B,C) chest CT multiplanar reconstructions. The cross-registration line in the axial reconstruction shows the same level of sagittal reconstruction, and single black arrow is IVC-RA junction; (D) chest CT coronal reconstruction (double-sided arrow is distance from carina to IVC-RA junction). AA, ascending aorta; DA, descending aorta; RA, right atrium; VBU, vertebral body unit; IVC, inferior vena cava; CXR, chest radiography.

Table 1 Measured distances and distances from carina to IRJ

\begin{tabular}{|c|c|c|c|c|c|}
\hline & Carina-IRJ (mm) & VBU(S) (mm) & $\operatorname{VBU}(\mathrm{C})(\mathrm{mm})$ & 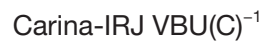 & Carina-IRJ VBU(S) $)^{-1}$ \\
\hline \multicolumn{6}{|l|}{ Gender } \\
\hline Male & $80.9(9.4)(56.5-112.0)$ & 24 (1.9) (19.7-30.3) & $22.8(1.4)(19.4-28.0)$ & $3.54(0.4)(2.6-5.0)$ & $3.42(0.5)(2.4-5.0)$ \\
\hline Female & 76.7 (9.0) (54.4-99.4) & 22.1 (1.5) (18.4-25.8) & 20.9 (1.2) (17.7-24.1) & $3.69(0.4)(2.5-5.1)$ & $3.49(0.5)(2.4-5.1)$ \\
\hline
\end{tabular}

Values are means (SDs) with ranges in parentheses (where applicable). Carina-IRJ, the distance between carina and IVC-RA junction; VBU(S), vertebral body unit on CXR; VBU(C), vertebral body unit on chest CT; Carina-IRJ VBU(C) ${ }^{-1}$, the distance between carina and IVCRA junction in VBU on chest CT; Carina-IRJ VBU(S) ${ }^{-1}$, the distance between carina and IVC-RA junction in VBU on chest radiograph; VBU, vertebral body unit; IVC, inferior vena cava; CXR, chest radiography.

106 males and 76 females, and the patient age varied from 26 to 92 years (median, 62 years). The median height was $163 \mathrm{~cm}$ (range, 144-186 cm), median weight was $61 \mathrm{~kg}$ (range, $40-106 \mathrm{~kg}$ ), and median BMI was $22.8 \mathrm{~kg} / \mathrm{m}^{2}$ (range, $16.2-34.2 \mathrm{~kg} / \mathrm{m}^{2}$ ). The measured distance and the distance from the carina to the IRJ in VBUs are summarized in
Table 1. The inter-rater correlation coefficients ( $\mathrm{r}$ ) for the distance from the carina to the IRJ and the height of vertebral bodies measured on chest CT and CXR were 0.90 , 0.83 and 0.890 , respectively $(\mathrm{P}<0.001)$.

The correlation between the distance from carina to IRJ in VBUs on CT and on CXR was high $(\mathrm{r}=0.930, \mathrm{P}<0.001)$ 


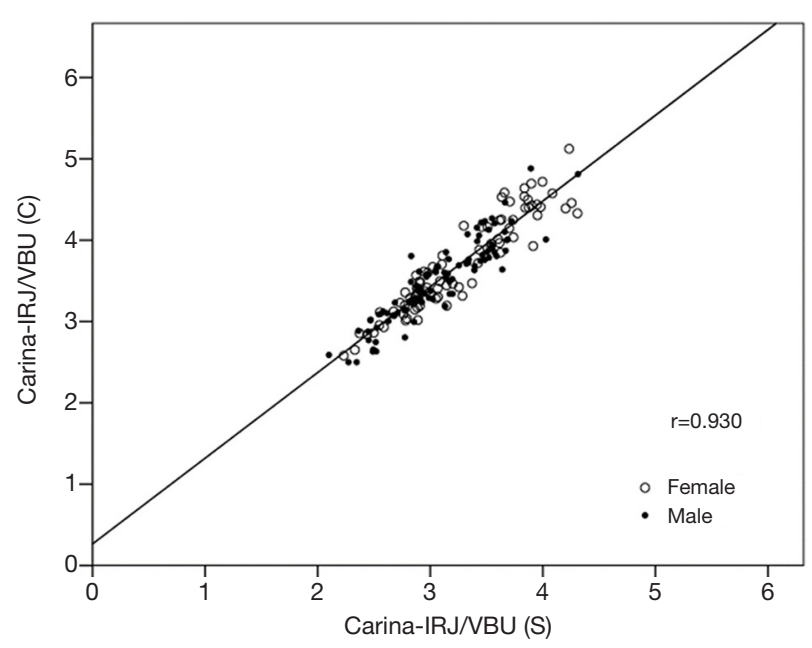

Figure 2 The relationship between the distance from the carina to the IRJ in VBUs on CXR [Carina-IRJ $\operatorname{VBU}(S)^{-1}$ ] and on chest CT [Carina-IRJ VBU $(C)^{-1}$ ]. VBU, vertebral body unit; CXR, chest radiography; Carina-IRJ $\mathrm{VBU}(\mathrm{C})^{-1}$, the distance between carina and IVC-RA junction in VBU on chest CT; Carina-IRJ VBU(S) ${ }^{-1}$, the distance between carina and IVC-RA junction in VBU on chest radiograph.

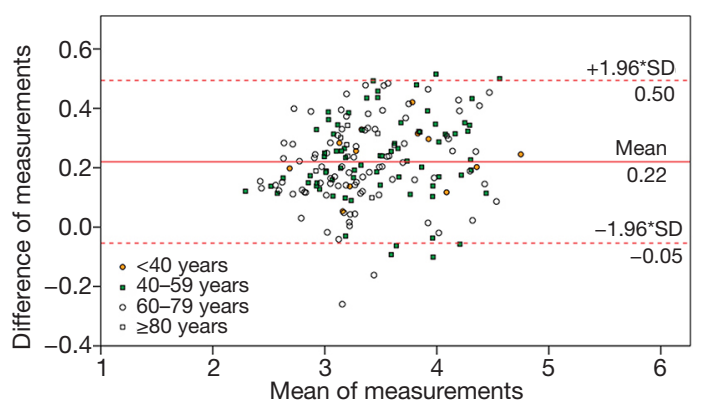

Figure 3 Bland-Altman plot of the agreement between VBU measurements using chest CT and CXR. Dashed lines are the limits of agreement, which are -0.05 and 0.5 . VBU, vertebral body unit; CXR, chest radiography.

(Figure 2). The Bland-Altman plot between measurements (VBU on CT minus VBU on CXR) showed 95\% limits of agreement between -0.05 and 0.5 (Figure 3).

Univariate analysis showed weak but significant correlations between the distance from the carina to the IRJ in VBUs on CXR and weight $(\mathrm{r}=-0.179, \mathrm{P}<0.001)$ and $\mathrm{BMI}(\mathrm{r}=-0.213, \mathrm{P}<0.001)$ (Table 2). Male, age and BMI were significantly associated with the distance from the carina to the IRJ on CXR $(\mathrm{P}=0.005,0.025$ and 0.001 , respectively) in multiple linear regression analysis (Table 3).

\section{Discussion}

One of the factors that play an important role in ECMO success is cannula, which provides an interface between ECMO circuit and patient. In general, the cannula is designed to maximize blood flow while minimizing damage to the blood itself. This depends on the material of a cannula, the surface coating of the cannula. And, the side holes for sufficient venous drainage and the double lumen cannula for simultaneous drainage and reinfusion are considered. However, the cannula length is determined by the type of ECMO circuit used (7). Also, Martucci and co-workers (8) suggested that using shorter and more biocompatible cannula during $\mathrm{V}-\mathrm{V}$ ECMO would further reduce transfusion and cost.

If the drainage cannula tip position is not properly located in the classic configuration of V-V ECMO, the increased possibility of recirculation or other problems may arise (2). Therefore, proper drainage cannula tip location is important. The confirmation of optimal cannula tip position was made by echocardiography in general (4), which is dependent on the operator and is inconvenient for the patients when performed.

Spine was used as a ruler to determine optimal CVC tip positioning $(5,9)$. The spine is minimally affected by geometric magnification and is adaptive to somatic growth, which aids optimal catheter tip positioning (5). Radiologic landmarks for estimating the optimal drainage cannula tip position in the classic configuration of $\mathrm{V}-\mathrm{V}$ ECMO have not been investigated. The carina and thoracic spine have been demonstrated as a landmark and internal ruler with easily visible in CXR $(5,9)$. As an internal ruler, VBU is a measurement relative to the body shape, not a fixed measurement, and we see this as a great advantage.

In the present study using thoracic spine as an internal ruler, the distance from carina to IRJ was $79.2 \mathrm{~mm}$ on chest CT and the position of the IRJ were 3.6 VBUs and 3.4 VBUs below the carina on chest CT and CXR. BlandAltman plot supported this correlation and in terms of the distance from carina to IRJ in VBU by CXR. So, if bedside chest $\mathrm{X}$-ray will be performed before the cannula position is fixed to the skin during the placement of ECMO cannula, our results may be helpful in determining the proper cannula tip position.

In the present study, the carina to IRJ distance was statistically significant correlated with height and BMI 
Table 2 Correlation analysis between continuous variables

\begin{tabular}{|c|c|c|c|c|c|c|c|c|c|c|}
\hline Variables & \multicolumn{2}{|c|}{ Carina-IRJ } & \multicolumn{2}{|c|}{$\operatorname{VBU}(\mathrm{C})$} & \multicolumn{2}{|c|}{ VBU(S) } & \multicolumn{2}{|c|}{ 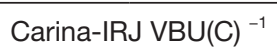 } & \multicolumn{2}{|c|}{ Carina-IRJ VBU(S) $^{-}$} \\
\hline Age & -0.076 & 0.044 & -0.065 & 0.075 & -0.057 & 0.355 & -0.038 & 0.322 & -0.048 & 0.2 \\
\hline Height & 0.348 & $<0.001$ & 0.672 & $<0.001$ & 0.522 & $<0.001$ & -0.052 & 0.10 & -0.022 & 0.544 \\
\hline Weight & 0.016 & 0.765 & 0.419 & $<0.001$ & 0.318 & $<0.001$ & -0.254 & $<0.001$ & -0.179 & $<0.001$ \\
\hline
\end{tabular}

Carina-IRJ, the distance between carina and IVC-RA junction; VBU(C), vertebral body unit on chest CT; VBU(S), vertebral body unit on CXR; Carina-IRJ VBU $(C)^{-1}$, the distance between carina and IVC-RA junction in VBU on chest CT; Carina-IRJ VBU(S) ${ }^{-1}$, the distance between carina and IVC-RA junction in VBU on chest radiograph; BMI, body mass index; VBU, vertebral body unit; IVC, inferior vena cava; CXR, chest radiography.

Table 3 Multiple linear regression analysis for the Carina-IRJ $\operatorname{VBU}(\mathrm{S})^{-1}$

\begin{tabular}{lcccc}
\hline \multirow{2}{*}{ Variables } & Coefficient & \multicolumn{2}{c}{$95 \% \mathrm{Cl}$} & \multirow{2}{*}{$\mathrm{P}$} \\
\cline { 3 - 4 } & & Lower & Upper & \\
\hline Constant & 4.627 & 4.028 & 5.226 & $<0.001$ \\
Gender (ref: female) & & & & \\
Male & -0.214 & -0.363 & -0.066 & 0.005 \\
Age & -0.006 & -0.012 & -0.001 & 0.025 \\
BMl & -0.034 & -0.055 & -0.014 & 0.001 \\
\hline
\end{tabular}

Carina-IRJ VBU(S) ${ }^{-1}$, the distance between carina and IVC-RA junction in VBU on chest radiograph; BMI, body mass index; IVC, inferior vena cava; VBU, vertebral body unit.

$(\mathrm{r}=0.348, \mathrm{r}=-0.179, \mathrm{P}<0.001, \mathrm{P}<0.001$, respectively). The distance had a weak but negative correlated with age $(\mathrm{r}=-0.076, \mathrm{P}=0.044)$. And, BMI was significantly associated with the distance from the carina to the IRJ on CXR $(\mathrm{P}=0.001)$ in multiple linear regression analysis. Recently, each year the aging population increase at a faster rate (10). Body changes with age impacts on the structure of the heart and thoracic cavity. We assume that many factors such as left ventricular hypertrophy, narrowing of intervertebral disk space, degenerative changes of vertebrae, and decreased lung elasticity impact on the position of the IRJ below the carina (10-12).

In our study, The VBU was $23.3 \mathrm{~mm}$ (SD 2.1) on CXR. However, in the previous study of Korean patients (9), the VBU was $22.6 \mathrm{~mm}$ (SD 2.1). Although we are studying patients in the same region, we believe that such differences are due to different sample size and study groups. In addition, the VBU on CXR and on chest CT had significant association with height and weight $(\mathrm{P}<0.001$, $\mathrm{P}<0.001$, respectively). Previous a random study comprised 829 patients showed that there was a moderate positive correlation between body height and vertebral height from T4 to L4 vertebrae (13). More-over, Salamon and others reported that disk height was positively correlated with body length and weight from T12 to S1 vertebrae (14). Hence, we thought that our results are in line with those of previous studies $(13,14)$.

Race-based difference in VBU is important considerations in our study. Previous studies $(15,16)$ reported significant differences in related vertebra parameter such as height between races. Although we did not study the relationship between race and $\mathrm{VBU}$, we assumed that racial differences would have a significant impact on VBU. Therefore, we recommend that a large-scale clinical trial be conducted to overcome the limitations of our study.

There were statistically significant differences in VBU on CXR and chest CT between men and female $(\mathrm{P}<0.001$, $\mathrm{P}<0.001$, respectively). Also, on multiple analysis, significant correlation was found between the position of the IRJ below the carina on CXR and male in the present study $(\mathrm{P}=0.005)$. Lowery and others (10) reported that after 40 years, kyphosis angle of the thoracic spine begins to increase more rapidly in women than men. And, bone loss in menopause women is associated with age (17). Therefore, we believe that this gender disparity is due to the postmenopausal estrogen deficiency and more progression of osteoporosis in women.

There are two V-A ECMO configurations: central and peripheral. In peripheral V-A ECMO, drainage cannula tip is mainly located in the infrahepatic IVC or in the RA (3). So, our study will help to ensure proper drain cannula tip position in peripheral V-A ECMO as in V-V ECMO. 


\section{Conclusions}

The present study has limitation. This was conducted at a single institution in a city with more than one million people in Korea and the results may not be generalized. Therefore, multicenter study should be conducted to obtain strong scientific evidence.

In conclusion, although this study was limited to Asians, this study showed that the mean position of the IRJ was estimated to be 3.4 VBUs below the carina on supine CXR. This may be useful if applied to the assessment of ECMO cannula tip positions.

\section{Acknowledgments}

We acknowledge Mi Hyeon Jin's assistance with statistical analysis (Department of Biostatics, Samsung Changwon Hospital, Sungkyunkwan University School of Medicine).

\section{Footnote}

Conflicts of Interest: The authors have no conflicts of interest to declare.

Ethical Statement: The authors are accountable for all aspects of the work in ensuring that questions related to the accuracy or integrity of any part of the work are appropriately investigated and resolved. The institutional review board approved this retrospective study and written informed consents were waived.

\section{References}

1. Thiagarajan RR, Barbaro RP, Rycus PT, et al. Extracorporeal Life Support Organization Registry International Report 2016. ASAIO J 2017;63:60-7.

2. Abrams D, Bacchetta M, Brodie D. Recirculation in Venovenous Extracorporeal Membrane Oxygenation. ASAIO J 2015;61:115-21.

3. Jayaraman AL, Cormican D, Shah P, et al. Cannulation strategies in adult veno-arterial and veno-venous extracorporeal membrane oxygenation: techniques, limitations, and special considerations. Ann Card Anaesth 2017;20:S11-8.

4. Banfi C, Pozzi M, Siegenthaler N, et al. Veno-venous extracorporeal membrane oxygenation: cannulation techniques. J Thorac Dis 2016;8:3762-73.

5. Baskin KM, Jimenez RM, Cahill AM, et al. Cavoatrial Junction and Central Venous Anatomy: Implications for Central Venous Access Tip Position. J Vasc Interv Radiol
2008;19:359-65.

6. Terpenning $S$, White CS. Imaging pitfalls, normal anatomy, and anatomical variants that can simulate disease on cardiac imaging as demonstrated on multidetector computed tomography. Acta Radiol Short Rep 2015;4:2047981614562443.

7. Kohler K, Valchanov K, Nias G, et al. ECMO cannula review. Perfusion 2013;28:114-24.

8. Martucci G, Panarello G, Occhipinti G, et al. Impact of cannula design on packed red blood cell transfusions: technical advancement to improve outcomes in extracorporeal membrane oxygenation. J Thorac Dis 2018;10:5813.

9. Song YG, Byun JH, Hwang SY, et al. Use of vertebral body units to locate the cavoatrial junction for optimum central venous catheter tip positioning. Br J Anaesth 2015;115:252-7.

10. Lowery EM, Brubaker AL, Kuhlmann E, et al. The aging lung. Clin Interv Aging 2013;8:1489-96.

11. Pugh KG, Wei JY. Clinical implications of physiological changes in the aging heart. Drugs Aging 2001;18:263-76.

12. Mahler DA, Rosiello RA, Loke J. The aging lung. Clin Geriatr Med 1986;2:215-25.

13. Skowrońska-Jóźwiak E, Płudowski P, Karczmarewicz E, et al. Effect of sex, age, and anthropometric parameters on the size and shape of vertebrae in densitometric morphometry. Pol Arch Med Wewn 2010;120:189-96.

14. Salamon NM, Van Langenhove C, Verstraete KL. Height of lumbar disc and vertebral body: what is the relation with body mass index, subcutaneous fat thickness, body weight, length and age? Vienna: Poster presented at European Society of Radiology, 2017.

15. Ning L, Song LJ, Fan SW, et al. Vertebral heights and ratios are not only race-specific, but also gender-and region-specific: establishment of reference values for mainland Chinese. Arch Osteoporos 2017;12:88.

16. Lau EM, Chan HH, Woo J et al. Normal ranges for vertebral height ratios and prevalence of vertebral fracture in Hong Kong Chinese: a comparison with American Caucasians. J Bone Miner Res 1996;11:1364-8.

17. Ahlborg HG, Johnell O, Turner $\mathrm{CH}$, et al. Bone loss and bone size after menopause. N Engl J Med 2003;349:327-34.

Cite this article as: Kim DS, Song YG, Kim YH, Lee MO. The position of the inferior vena cava-right atrium junction on simple radiography: implication for optimal veno-venous extracorporeal membrane oxygenation cannula tip position. J Thorac Dis 2019;11(9):3881-3886. doi: 10.21037/jtd.2019.09.10 